\title{
El papel de los actores de la cuádruple hélice en el emprendimiento tecnológico de Tamaulipas
}

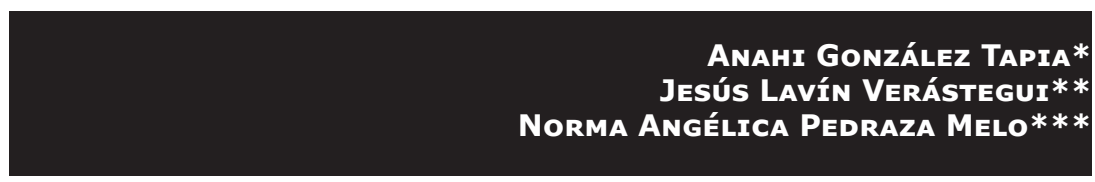

\section{RESUMEN}

El objetivo de esta investigación es conocer el papel que desempeñan los actores de la cuádruple hélice en el emprendimiento tecnológico en Tamaulipas, con la finalidad de profundizar en el entendimiento de este fenómeno desde la perspectiva de los diferentes agentes. Este estudio se aborda desde el método cualitativo, con la realización de entrevistas, para explorar el trabajo y las relaciones que se generan para crear un entorno óptimo para esta actividad. Los principales hallazgos sugieren que, aunque existe vinculación entre los agentes en diferentes modalidades, aún es necesario mejorar los esfuerzos para superar los desafíos a través de estrategias específicas.

Palabras clave: emprendimiento tecnológico, cuádruple hélice, innovación, Tamaulipas.

Clasificación JEL: L26, O39.

* Estudiante del Doctorado en Ciencias Administrativas de la Universidad Autónoma de Tamaulipas, México. ORCID: https://orcid.org/0000-0001-9429-1360. Correo electrónico: anahi. glz@hotmail.com

** Director del Centro de Innovación y Transferencia de Conocimiento, Universidad Autónoma de Tamaulipas, México. ORCID: https://orcid.org/0000-0002-8590-664X. Correo electrónico: jelavin@docentes.uat.edu.mx

*** Profesor de Tiempo Completo, Universidad Autónoma de Tamaulipas, México. ORCID: https://orcid.org/0000-0001-9566-2880. Correo electrónico: napedraza@docentes.uat.edu.mx 


\section{ABStRACT}

\section{The Role of the Quadruple Helix Actors in the Technological Entre- preneurship of Tamaulipas}

The aim of this investigation is to know the role played by the actors of the quadruple helix in the technological entrepreneurship in Tamaulipas, to deepen the understanding of this phenomenon from the perspective of the different agents. This research is approached from the qualitative method, using interviews to explore the work and relationships that are carried out to create an optimal environment for this activity. The main findings suggest that, although there is a link between the agents in different modalities, it is still necessary to improve efforts to overcome the challenges through specific strategies.

Keywords:technological entrepreneurship, quadruple helix, innovation, Tamaulipas.

JEL Classification:L26, O39.

\section{INTRODUCCIÓN}

En los últimos años se ha observado un elevado interés por estudiar temas relacionados con la innovación, emprendimiento y desarrollo económico como resultado de los cambios sociales, económicos y culturales que enfrenta el mundo y que impactan de manera directa el bienestar social y la sostenibilidad económica de las naciones (Urbano et al., 2018; Zahra y Wright, 2016). Específicamente, se analiza el tópico de colaboración entre diversos actores que, de acuerdo con Guerrero y Urbano (2017), propicia un entorno de conocimiento intensivo e ideal para realizar actividades innovadoras y empresariales, como un medio para generar crecimiento en economías emergentes como México, que vive una transición hacia una economía basada en el conocimiento.

En este contexto nace el modelo de la triple hélice, que pretende explicar el desempeño estructural de las relaciones universidad-industria-gobierno (Etzkowitz y Leydesdorff, 1995; Leydesdorff, 2012) y que antecede a la incorporación de un cuarto agente: la sociedad civil, que aporta la visión del consumidor de las innovaciones empresariales (Afonso et al., 2012). En este sentido, en México se habla de la colaboración triple hélice como una actividad recurrente pero inconsistente 
que, si bien ha sido promovida por diversos agentes, sigue sin lograr los resultados esperados, a pesar de los esfuerzos del gobierno por generar políticas públicas que permitan el buen desarrollo de ciencia, tecnología e innovación (Bautista, 2014).

El avance más notorio en los últimos años se logró mediante la reforma a la Ley de Ciencia y Tecnología y la Ley Federal de Responsabilidades Administrativas de los Servidores Públicos, en donde se da pie al uso de conocimientos adquiridos por académicos en su trayectoria con fines comerciales, se impulsa la transferencia de conocimiento y se busca un cambio en el desempeño de indicadores de ciencia, tecnología e innovación. Sin embargo, no existe información oficial que exponga los resultados de esta política pública. Por otra parte, aunque el gasto gubernamental en ciencia y tecnología ha tenido un ligero aumento en la última década, aún es bajo el porcentaje del Producto Interno Bruto (PIB) destinado a esta actividad (Dosal et al., 2011).

Respecto al PIB que los países de América Latina y el Caribe con ingreso mediano alto aportaron a la investigación y desarrollo en 2017, México, con $0.32 \%$, se situó debajo de Costa Rica (0.42\%), Cuba $(0.43 \%)$ y Argentina $(0.54 \%)$, y superó a Colombia ( $0.24 \%)$, Paraguay $(0.14 \%)$ y Perú $(0.12 \%)$. Además, el mismo nivel de gasto se encuentra dentro del rango de otros con el mismo tipo de ingresos de Europa y Asia central, por ejemplo, Georgia (0.29\%) y Montenegro $(0.34 \%)$ (Banco Mundial, 2020). Derivado de esto, se percibe la necesidad de concientizar sobre la importancia de estas actividades para el desarrollo.

En este sentido, en el país se llevan a cabo eventos de iniciativa privada que fomentan la innovación tecnológica, como el Innovación Latam México, Expo Léon Technology \& Innovation México, o Jalisco Talent Land, en donde se presentan paneles de debate, mesas redondas, conferencias, talleres y oportunidades de creación de redes de colaboración. Además, se encuentran vigentes dos programas federales para fomentar el desarrollo tecnológico: el Programa Estratégico Nacional de Tecnología e Innovación Abierta (Penta), que apoya a entidades públicas o privadas que desarrollen proyectos de innovación tecnológica abierta y de impacto social (Conacyt, 2019), y el Programa para el Desarrollo de la Industria del Software y la Innovación (Prosoft), que promueve el desarrollo y la adopción de tecnologías de información y la innovación en los sectores estratégicos del país (Secretaría de Economía, 2016). 
En Tamaulipas se ha implementado el programa Creciendo con tu empresa, el Certamen Estatal de Creatividad e Innovación Tecnológica (CECIT) y otros servicios dirigidos a la creación de una cultura de registro de propiedad industrial del Consejo Tamaulipeco de Ciencia y Tecnología (Cotacyt). A pesar de esto, se han identificado cuatro limitantes principales para la comercialización de innovación en el país: asimetría de información entre empresarios o emprendedores, fallas de coordinación o vigilancia tecnológica, bienes públicos o apropiabilidad, y externalidades (Dosal et al., 2011). En la asimetría de información, por ejemplo, no todos los participantes de una actividad cuentan con la misma información, lo que desequilibra las oportunidades de éxito en la toma de decisiones. Algunos empresarios o emprendedores poseen más y mejor información que otros; esto puede derivar en proyectos de negocios de baja calidad e inhabilidad de los innovadores para explotar todas las oportunidades de su plan de negocios, así como una actitud negativa hacia inversionistas externos (Zavatta, 2008). Por otra parte, la falta de vigilancia tecnológica o coordinación puede restringir la visualización de oportunidades de acceso a financiamiento (Dosal et al., 2011).

En el caso de la apropiabilidad de bienes públicos para innovar, se debe considerar que su consumo no reduce la cantidad disponible para las demás personas, por tanto, no se puede excluir o limitar su uso; mientras que en las externalidades cabe cualquier otro efecto que ocasione la producción o consumo de un bien en el público general (Dosal et al., 2011). En consecuencia, se deben aplicar estrategias para disminuir el impacto de estas limitantes, no sólo desde la administración pública, sino desde la visión de los empresarios innovadores para potencializar sus oportunidades de éxito.

Aunado a lo anterior, la actividad emprendedora de los jóvenes mexicanos se ha visto afectada por el entorno socioeconómico, que ha ocasionado que ésta sea vista como una forma de sobrevivencia más que para generar negocios prósperos y sostenibles, lo que les permitiría explorar más a fondo su motivación personal y capacidades físicas e intelectuales (Canales et al., 2017). En este sentido, Moreno y Espíritu (2010) sostienen que la triple hélice impulsa la creación de empresas mediante la educación, el soporte financiero y el apoyo de la experiencia empresarial, por lo que es relevante incorporar su visión en estrategias 
y políticas públicas orientadas al crecimiento económico a través del emprendimiento, la creación de empleos formales y la innovación.

Derivado de ello, el objetivo de esta investigación es conocer el papel que desempeña cada actor de la cuádruple hélice en el emprendimiento tecnológico en Tamaulipas. El documento se integra por una sección introductoria que expone la importancia y situación actual del fenómeno; posteriormente se describen las características del modelo de la cuádruple hélice; en la siguiente sección se habla del origen y estudio del emprendimiento tecnológico, seguido de los trabajos sobre la relación de ambas variables. En otro apartado se abordan la estrategia metodológica y se presentan los hallazgos, y por último, se muestran las reflexiones finales y futuras líneas de investigación.

\section{CARActerísticas del MOdelo de LA CuÁdRUPLE hÉliCE}

Previo a la cuádruple hélice existieron modelos teóricos que buscaron explicar las relaciones entre diversos agentes, como el lineal de innovación o modo 1, el modelo interactivo o modo 2, el triángulo de Sábato y la triple hélice (Castillo et al., 2014) que, como se mencionó en el apartado anterior, fue la base para descubrir nuevas relaciones con otros actores, en donde el desarrollo de novedosas tecnologías, productos y servicios ya no era sólo de interés de la empresa, sino que ahora se invita a participar a otros subsistemas (sociedad) en contextos diferentes (sustentabilidad), para lo que se plantean nuevos modelos que incluyen una cuarta, quinta y $\mathrm{N}$ hélices para su descripción (Figura 1) (Afonso et al., 2012; Carayannis y Campbell, 2009; Leydesdorff, 2012), y que se abrirían a esquemas más complejos como la innovación abierta (Parveen et al., 2015) y ecosistemas de innovación (Arenal et al., 2020).

FIGURA 1

ANTECEDENTES DEL MODELO CUÁDRUPLE HÉLICE

\begin{tabular}{|c|c|c|c|c|c|}
\hline $\begin{array}{c}\Delta \text { Modelo } \\
\text { lineal o } \\
\text { modo } 1\end{array}$ & $\begin{array}{l}\Delta \text { Modelo } \\
\text { interactivo } \\
\text { o modo } 2\end{array}$ & $\begin{array}{r}\Delta \text { Triángulo } \\
\text { de Sábato } \\
\text { (Sábato y }\end{array}$ & $\begin{array}{c}\Delta \text { Sistemas de } \\
\text { innovación } \\
\text { (Freeman }\end{array}$ & $\begin{array}{l}\Delta \text { Triple } \\
\text { hélice } \\
\text { (Etzkowitzy }\end{array}$ & $\begin{array}{l}\Delta \text { Cuádruple, } \\
\text { quintuple y } \\
N \text { hélices }\end{array}$ \\
\hline $\begin{array}{r}\text { (Gibbons, et } \\
\text { al., 1994) }\end{array}$ & $\begin{array}{r}\text { (Rosenberg, } \\
\text { 1976; Kline y } \\
\text { Rosenberg, } \\
1986)\end{array}$ & $\begin{array}{r}\text { Botana, } \\
1968)\end{array}$ & $\begin{array}{r}1987 ; \\
\text { Lundvall, } \\
1985)\end{array}$ & $\begin{array}{r}\text { Leydesdorff, } \\
1995)\end{array}$ & $\begin{array}{l}\text { (Leydesdorff, } \\
\text { 2012) }\end{array}$ \\
\hline
\end{tabular}

Fuente: elaborado a partir de Castillo et al. (2014). 
Como se muestra en la Figura 1, los modelos que antecedieron a la cuádruple hélice son: el modelo lineal o modo 1, que se desarrolla de la academia hacia el sector productivo; se realza la actividad científica y se transmite mediante publicaciones académicas; el interactivo o modo 2 , que da un papel central a la empresa y se enfoca en la aplicabilidad y utilidad social de la investigación realizada, y el triángulo de Sábato, que busca identificar los actores en la colaboración entre el gobierno, el sector productivo y la infraestructura científico-tecnológica en el desarrollo regional. Por un lado, los sistemas de innovación permitieron integrar diversos agentes a un mismo nivel de interacción en las escalas regional y local. Por otro, el modelo de la triple hélice, desarrollado por Henry Etzkowitz y Loet Leydesdorff, en 1995, plantea múltiples esquemas de interacción para sus agentes (Castillo et al., 2014).

En cuanto al modelo cuádruple hélice, sugiere que la estructura económica de un país se basa en la colaboración de empresas, universidad, gobierno y sociedad civil dentro de una cultura cooperativa de intercambio de conocimientos en un contexto de redes de colaboración, relaciones simbióticas y asociaciones, que deriva en la generación continua de innovación (Afonso et al., 2012; Afzal et al., 2018; Carayannis y Campbell, 2009). Cada agente cumple con un papel específico en el desarrollo económico y a través de sus interrelaciones favorece la creación de una sociedad del conocimiento (González, 2019).

En el caso de la universidad y centros de transferencia de conocimiento, su importancia recae en la educación y capacitación emprendedora de los egresados, la transferencia de conocimiento y tecnología, así como la intención emprendedora de los estudiantes, que además integra otra parte significativa del ecosistema innovador: las infraestructuras tecnológicas, como laboratorios universitarios e instalaciones industriales de investigación y desarrollo (Afonso et al., 2012). Por otra parte, las empresas, así como los parques científicos y tecnológicos aportan la infraestructura técnica para la investigación y el desarrollo en las industrias de alta y media tecnología, la generación de nuevos productos y servicios técnicos, así como redes, asociaciones y comités para el emprendimiento (Galvão et al., 2017; González, 2019).

El gobierno provee apoyo financiero y el sistema regulatorio para promover la creación de vínculos universidad-empresas mediante políticas públicas e instituciones puente, como parques científicos e incubadoras de empresas; además, realiza inversión en educación, salud, 
infraestructura, servicios, regulaciones tecnológicas y de innovación para aumentar la producción de los insumos (Afonso et al., 2012). Por su parte, la sociedad civil crea un entorno competitivo y de emprendimiento regido por normas sociales y culturales creadas por la atención mediática al emprendimiento, al exigir productos y servicios innovadores y de más calidad (Afzal et al., 2018; Galvão et al., 2017). No obstante, la visión social se ha descuidado en la literatura científica y el discurso político, lo que ha causado un desafío para la formulación de objetivos y aplicación de métodos apropiados para los procesos participativos (Schütz et al., 2019).

En este mismo orden de ideas, da Costa et al. (2018) analizaron información de 135 artículos de investigación identificados en la base de datos de Web of Science que resaltan la triple, cuádruple y quíntuple hélice principalmente desde los enfoques de sistemas de innovación y redes y medios de generación de conocimiento; el método de estudio de caso es el que más predomina, así como la teoría referente a temas de innovación y capacidad de absorción, tanto regional como empresarial, cuyas organizaciones intermediarias del modelo son las incubadoras, los parques tecnológicos y las aceleradoras de negocios (Del Giudice et al., 2017; González, 2019; Miller et al., 2016b; Miller et al., 2016a).

Estos resultados concuerdan con los hallazgos de Galvão et al. (2019), quienes destacan cuatro líneas de investigación sobre el fenómeno: 1) las políticas de innovación y conocimiento; 2) universidades emprendedoras; 3) estrategia de innovación empresarial, y 4) actores de la triple hélice en innovación, conocimiento y desarrollo regional. Sugieren futuras investigaciones sobre los tipos de canales de conocimiento utilizados en las interacciones entre los agentes para analizar sus aspectos geográficos, así como las barreras a la cooperación universidades-industrias desde el punto de vista de los investigadores académicos, el papel del consumidor en la creación de modelos de incubación de negocios para satisfacer las necesidades no regionales y organizativas, los impactos de las relaciones entre las hélices de las economías, las sociedades y el medio ambiente (Galvão et al.,2019; McAdam et al., 2016).

Asimismo, trabajos más recientes han estudiado las plataformas digitales que juegan un papel de intermediario en la colaboración cuádruple hélice (Vallance et al., 2020), innovación sustentable regional a partir de este modelo (Bărbulescu y Constantin, 2019; Iqbal 
et al., 2018; Roman et al., 2020), derrama de conocimiento en ecosistemas emprendedores (Marques et al., 2020), desarrollo de ciudades inteligentes (Borghys et al., 2020; Suzic et al., 2020), y generación de estrategias de especialización inteligente (Hasche et al., 2019; Höglund y Linton, 2018).

Por otra parte, cabe destacar que recientemente se realizó la conferencia virtual "Triple hélice 2020", organizada por la Triple Helix Association (asociación con fines científicos y de alcance global) que se realiza anualmente desde 2009, en la que académicos, expertos y formuladores de políticas, interesados en las relaciones dinámicas de este modelo en el ecosistema de innovación, discutieron sobre los cambios en las políticas públicas, las expectativas ante la crisis actual, así como temas emergentes de investigación, como la universidad emprendedora socialmente responsable y emprendedores digitales, lo que refuerza los hallazgos indicados anteriormente (Asociación Triple Hélice, 2020). No obstante, si bien la investigación del fenómeno ha tomado una dirección hacia modelos cuádruple y quíntuple hélice, los problemas relacionados con las tres hélices principales continúan siendo los más estudiados (da Costa et al., 2018; Galvão et al.,2019).

\section{ORIGEN Y ESTUDIO DEL EMPRENDIMIENTO TECNOLÓGICO}

Los primeros indicios del concepto de emprendimiento tecnológico aparecen en La riqueza de las naciones de Adam Smith (1776), obra en la que se habla de las causas y consecuencias del avance tecnológico; posteriormente, Karl Marx (1867) sugiere que el conocimiento tecnológico explica la evolución socioeconómica de la sociedad capitalista; sin embargo, fue Schumpeter (1934) quien introdujo el concepto de innovación tecnológica en el mundo de la investigación científica (Benavides, 2004; Morgan, 1997). A partir de esto, el concepto se comenzó a desarrollar desde un enfoque clásico de innovación empresarial, considerado un proceso dinámico que involucra la perturbación del estado económico actual por el empresario, al representar la voluntad y la acción necesarias para innovar (Baumol, 1989; Fernández, 2015; Ferreira et al., 2016).

Si bien Schumpeter presentó el concepto de innovación tecnológica en la teoría económica, se atribuye al economista Rupert Maclaurin los estudios sistemáticos sobre este fenómeno; en las décadas de 1940 y 
1950 desarrolló las ideas de Schumpeter y las analizó como un proceso compuesto de varias etapas, a partir de lo cual propuso una teoría con este mismo nombre, que más tarde sería llamada "Modelo lineal de innovación" (Godin, 2008). Esta teoría sugiere que la innovación comienza a partir de un descubrimiento científico, que debe ser verificado mediante la investigación aplicada, para posteriormente desarrollar la tecnología, su fabricación y concluir con la comercialización del bien o servicio (López et al., 2009).

En este sentido, el emprendimiento tecnológico existe cuando los desarrollos en la ciencia o la ingeniería constituyen una oportunidad para el surgimiento de una empresa, mercado, clúster, industria o productos, por lo que Ferreira et al. (2016) lo definen como un proceso que implica el descubrimiento y creación, que impulsa oportunidades de negocios. Por tanto, se habla de este concepto como la identificación y aprovechamiento de oportunidades comerciales de alto potencial e intensivas en tecnología, que puede basarse en un avance revolucionario o evolutivo, y apuntar a un mercado existente o crear uno nuevo, ya sea para empresas emergentes independientes o establecidas (Mosey et al., 2017; Urbano et al., 2018; Xue y Klein, 2010).

Sobre la investigación del fenómeno, Ferreira et al. (2016) realizaron un estudio bibliométrico de 135 artículos sobre emprendimiento tecnológico en un periodo de 27 años (de 1986 a 2013); mediante análisis de redes de co-citas, encontraron los artículos más citados (Cuadro 1). Resalta que los estudios se concentran en Estados Unidos, Reino Unido y Países Bajos, por lo que concluyen que si bien el tema sigue siendo un campo teórico emergente en evolución, su estudio empírico podría contribuir a desarrollar políticas y estrategias que fomenten esta actividad.

\section{CUADRO 1}

ARTículos MÁS CITADOS SOBRE EMPRENDIMIENTO TECNOLÓGICO

\begin{tabular}{ccc}
\hline No. & Fuente & Citaciones \\
1 & Garud y Karn $\varnothing(2003)$ & 227 \\
2 & Dushnitsky y Lenox (2005) & 93 \\
3 & Venkataraman (2004) & 67 \\
\hline
\end{tabular}

Fuente: elaboradoa partir de Ferreira et al. (2016). 
En el artículo más citado, Garud y Karnøe (2003) estudiaron el emprendimiento tecnológico; sugieren que sus desarrollos son el resultado de la influencia de diferentes actores responsables de los caminos tecnológicos emergentes capaces de generar más conocimiento. Por su parte, Dushnitsky y Lenox (2005) se centraron en los beneficios potenciales del capital de riesgo corporativo para la innovación como un medio para generar iniciativas empresariales que juegan un papel fundamental en la estrategia de innovación de una empresa. Mientras que Venkataraman (2004) indica que los gobiernos intentan promover el espíritu empresarial mediante inyección de capital de riesgo, lo que desde su perspectiva promueve el emprendimiento de baja calidad.

De acuerdo con Ferreira et al. (2016), la investigación sobre el emprendimiento tecnológico se ha desarrollado desde tres enfoques principales: el institucional, que sugiere que la existencia de instituciones y el apoyo a la investigación son factores clave para esta actividad; el enfoque gubernamental y financiero, que argumenta que el desarrollo de empresas de alta tecnología es fundamental para la existencia de políticas de apoyo gubernamental, que a su vez fomentan esta práctica; y el ambiental, que sostiene que ciertos tipos de entornos tienen características únicas que impulsan el crecimiento de éste. Por su lado, Beckman et al. (2012) y Marín y Rivera (2014) unieron los enfoques en dos generales: el emprendimiento y la innovación basada en tecnología.

Por otra parte, Mosey (2016) asegura que la discusión sobre la conceptualización e importancia del emprendimiento tecnológico ha alcanzado su madurez en la literatura y ha evolucionado hacia la construcción de teoría para alentar y mejorar esta práctica en contextos específicos, para lo que propone seguir explorando las competencias individuales de inicio de estudiantes y exalumnos, aceleradoras, redes de colaboración y crowdsourcing (colaboración abierta), así como utilizar diferentes teorías para su explicación, como la evolutiva, difusión del conocimiento y ecosistemas empresariales, lo que se refleja en trabajos posteriores.

En cuanto a competencias individuales, Wheadon y Duval-Couetil (2018) llevaron a cabo una revisión de literatura sobre género y emprendimiento en tecnología, en donde exploraron los factores particulares y contextuales que mantienen el estatus simbólico de las mujeres en el campo; evidenciaron las barreras asociadas al acceso al capital finan- 
ciero y la participación social, tras lo que sugieren realizar estudios más profundos que permitan analizar las barreras que enfrentan las féminas en el ambiente y los factores que las sostienen.

De igual manera, sobre el contexto, Urbano et al. (2018) estudiaron cómo algunas capacidades dinámicas estratégicas y las condiciones ambientales de apoyo influyen en la configuración de las iniciativas de emprendimiento tecnológico. Identificaron el papel positivo de la orientación empresarial y la orientación al mercado de exportación, así como que las condiciones ambientales reguladoras mejoran, mientras que otras condiciones reguladoras y normativas retardan a la par la probabilidad de que una empresa nueva o establecida desarrolle iniciativas de emprendimiento tecnológico, por lo que proponen analizar el efecto de moderación de las condiciones ambientales de apoyo, además de considerar la necesidad y oportunidad de emprendimiento en el nuevo mundo económico, así como los movimientos de la actividad (intra) empresarial entre países y la intersección entre los niveles individual, organizacional y ambiental.

Aunado a ello, se han identificado estudios orientados a mejorar la enseñanza de emprendimiento tecnológico desde el punto de vista educativo; por ejemplo, Duval et al. (2020) proponen un modelo de curso para la educación empresarial en ciencia y tecnología que permita a los investigadores académicos desempeñar un papel más activo en la comercialización de su descubrimiento. Mientras que Kleine et al. (2019) plantean un modelo para el proceso de aprendizaje en emprendimiento tecnológico basado en los resultados de un estudio de caso de un programa de ingeniería que resalta la capacidad empresarial de la tecnología. Por tanto, los estudios más recientes de ambos fenómenos marcan la pauta hacia la aplicabilidad de las aportaciones para mejorar las prácticas en contextos específicos.

\section{LA CUÁDRUPLE HÉLICE Y SU RELACIÓN CON EL EMPRENDIMIENTO TECNOLÓGICO PARA EL DESARROLLO ECONÓMICO REGIONAL}

Como se mencionó en los apartados anteriores, la investigación de la cuádruple hélice y del emprendimiento tecnológico continúan siendo temas de interés, por lo que quedan muchas áreas de oportunidad para su estudio, como la relación entre ambas. Si bien se han identificado 
varios esfuerzos por relacionar el contexto regional (Autio et al., 2014), las redes de colaboración o el ecosistema innovador con el emprendimiento tecnológico, sólo tres trabajos de investigación han considerado los modelos de la triple y cuádruple hélice específicamente: el de Galvão et al. (2017), el de Guerrero y Urbano (2017), y el de Byankin y Burdakova (2019).

En primera instancia, Galvão et al. (2017) estudiaron el papel del emprendimiento en el desarrollo económico, basado en las dimensiones del modelo cuádruple hélice, en relación con las tres etapas de la economía definidas por el Monitor de Emprendimiento Global (GEM, por sus siglas en inglés): innovación, economías impulsadas por la eficiencia y factores. Mediante un análisis de datos secundarios de 58 países en 2015, encontraron que en las economías impulsadas por factores existe mayor influencia de la industria, mientras que en las economías impulsadas por la eficiencia hay homogeneidad entre las cuatro dimensiones; destacan sólo las variables "transferencia de investigación y desarrollo" e "intención empresarial", mientras que en las economías impulsadas por innovación, las empresas y el gobierno son las más importantes.

En segunda instancia, Guerrero y Urbano (2017) analizaron los efectos producidos por los vínculos de las empresas con otras empresas, universidades y gobierno sobre su desempeño innovador, así como los efectos de moderación formados cuando tienen una orientación de alto crecimiento. Con base en datos de sección transversal de 19 188 compañías mexicanas entrevistadas de 2006 a 2012, encontraron que las empresas sí colaboran con cada actor de la triple hélice, lo que produce un efecto positivo en su desempeño innovador y se refuerza cuando tiene una orientación de alto crecimiento, mientras el contexto socioeconómico mostró un impacto negativo.

En tercera instancia, Byankin y Burdakova (2019), con base en un estudio de caso y revisión de literatura, desarrollaron propuestas para formar competencias de emprendimiento tecnológico en instituciones de educación superior, basándose en el modelo de la triple hélice; una de ellas sugiere incluir módulos específicos orientados a proyectos en la parte del plan de estudios de capacitación para los principales programas educativos, e implementar el módulo opcional interdisciplinario "emprendimiento". 
Con base en sus limitaciones, estos estudios proponen, para futuras líneas de investigación, reforzar los modelos propuestos mediante el uso de técnicas, como el análisis multinivel, para medir el impacto regional, o un estudio longitudinal, para observar la evolución de las economías a lo largo del tiempo, además del uso de otras bases de datos para analizar distintas variables en distintos países (Galvão et al., 2017; Guerrero y Urbano, 2017). Derivado de estas propuestas, las insuficiencias del estudio de la relación de la cuádruple hélice y el emprendimiento tecnológico, así como la problemática en México, surge la necesidad de profundizar en este fenómeno desde un enfoque cualitativo, lo que deriva en la pregunta de investigación: ¿cuál es el papel que desempeñan cada uno de los actores de la cuádruple hélice en el emprendimiento tecnológico en Tamaulipas? (Hamui, 2016).

\section{ESTRATEgIA METOdOLÓgICA}

Para dar respuesta a la pregunta establecida, se llevó a cabo una investigación cualitativa de diseño fenomenológico, lo que permitió recabar información desde la realidad de los sujetos de estudio (Cadena-Iñiguez et al., 2017; Riese, 2019; Salgado, 2007). Asimismo, la investigación es de tipo exploratorio y descriptivo, ya que pretende ahondar en un evento no estudiado que, como se mencionó, incluye temas de desarrollo en la investigación científica y que además permite detallar las propiedades y características de los hallazgos (Hernández et al., 2003; Saunders et al., 2009).

En este sentido, se diseñó una entrevista semiestructurada para recolectar información y cumplir con el objetivo de la presente investigación (Aarsand y Aarsand, 2018; Saunders et al., 2009), la cual fue tratada mediante análisis de contenido (Díaz, 2018), con atención al rigor metodológico (Castillo y Vásquez, 2003) a través de los siguientes criterios de validez y confiabilidad utilizados en el proceso: coherencia metodológica para garantizar la congruencia entre la pregunta de investigación y los componentes del método; una muestra cualitativa apropiada formada por participantes clave en el tema; un proceso de recolección de información y análisis bien ejecutados (Moral, 2006; Morse et al., 2002; Plaza et al., 2017).

Con referencia a lo anterior, se habla de las entrevistas semiestructuradas como un método de recolección de información para la inves- 
tigación cualitativa, a través de una serie de preguntas a un sujeto de estudio, a fin de conocer su perspectiva sobre un fenómeno en específico (Aarsand y Aarsand, 2018; Saunders et al., 2009; Symon y Cassell, 2004). Por tanto, el tipo de entrevista seleccionado fue el fenomenológico, ya que hace énfasis en la necesidad de que el investigador deje a un lado sus presuposiciones sobre el fenómeno en estudio (Salgado, 2007).

El proceso de construcción de las entrevistas inició con la definición de las preguntas a partir del objetivo de investigación (Hamui, 2016), con base en la literatura existente. Posteriormente se realizó la guía para la audiencia (Symon y Cassell, 2004), integrada por tres secciones específicas; en la primera se situó la presentación del entrevistador, el propósito, una breve contextualización de los principales conceptos, así como los términos de confidencialidad y uso de información; en la segunda se incluyeron las preguntas base (Cuadro 2); y en la última se agregó el diálogo de conclusión, agradecimiento y comentarios (Aarsand y Aarsand, 2018; Saunders et al., 2009).

\section{CUADRO 2}

PREGUNTAS DE LA ENTREVISTA

\begin{tabular}{|c|c|}
\hline Pregunta & Respaldo teórico \\
\hline $\begin{array}{l}\text { 1. ¿Cómo percibe usted el desarrollo del emprendimiento tecnológico en } \\
\text { Tamaulipas? ¿Por qué? ¿Qué desafíos identifica para fomentarlo? ¿Qué } \\
\text { estrategias observa necesarias para impulsarlo? }\end{array}$ & $\begin{array}{l}\text { Canales et al. (2017), } \\
\text { Dosal et al. (2011), } \\
\text { Sánchez et al. (2014) }\end{array}$ \\
\hline $\begin{array}{l}\text { 2. ¿Cómo impulsa sus actividades el emprendimiento tecnológico en el } \\
\qquad \text { estado? }\end{array}$ & $\begin{array}{l}\text { Guerrero y Urbano (2017), } \\
\text { Urbano et al. (2018) }\end{array}$ \\
\hline $\begin{array}{l}\text { 3. ¿De qué manera se vincula con otros actores (por ejemplo, con las } \\
\text { universidades, el sector empresarial, el gobierno y la sociedad civil) para poder } \\
\text { llevar a cabo estas actividades de impulso? }\end{array}$ & $\begin{array}{l}\text { Bautista (2014), } \\
\text { Guerrero y Urbano (2017) }\end{array}$ \\
\hline $\begin{array}{l}\text { 4. ¿Cómo ha beneficiado al emprendimiento tecnológico estas actividades de } \\
\qquad \text { vinculación? }\end{array}$ & $\begin{array}{l}\text { Dosal et al. (2011), } \\
\text { Malecki (2018) }\end{array}$ \\
\hline $\begin{array}{l}\text { 5. ¿Qué recomendaría implementar para fomentar el emprendimiento } \\
\text { tecnológico en Tamaulipas? }\end{array}$ & $\begin{array}{l}\text { Dosal et al. (2011), } \\
\text { Malecki (2018) }\end{array}$ \\
\hline
\end{tabular}

Fuente: elaborado a partir de autores indicados en respaldo teórico.

Como se observa en el Cuadro 2, los estudios base para construir las preguntas de la entrevista se realizaron en América Latina y México; sin embargo, el más cercano al contexto regional es de Sánchez et al. (2014), que si bien no abordan directamente la cuádruple hélice y su 
relación con el emprendimiento tecnológico, están implícitos en sus resultados. En este tenor, la primera pregunta se elaboró para verificar los hallazgos de Canales et al. (2017), Dosal et al. (2011) y Sánchez et al. (2014), quienes estudiaron el contexto mexicano y encontraron que el emprendimiento surge más como una forma de sobrevivencia que para explotar el entorno y las capacidades de los emprendedores, además de que la inversión se da más en sectores de baja tecnología.

Aunado a lo anterior, los autores encontraron algunos desafíos en el emprendimiento en México, por ejemplo, incentivar de manera intensiva proyectos empresariales mediante políticas públicas y programas de financiamiento estimula negocios con bajo aporte a la innovación y pocas probabilidades para sobrevivir a largo plazo, así como procesos burocráticos ineficientes, tiempos de espera extremos y falta de conocimientos de algunos administradores sobre los requisitos para realizar convenios o proyectos de investigación colaborativa, además de un déficit de inversión pública en infraestructura para la actividad innovadora, el uso de tecnologías obsoletas y la falta de especialización universitaria (Canales et al., 2017; Dosal et al., 2011; Sánchez et al., 2014).

Por otra parte, con la segunda pregunta se buscó enriquecer los hallazgos de Guerrero y Urbano (2017), quienes identificaron que las empresas mexicanas cooperan intraemprendedoramente (con sus padres), comercialmente (con otras empresas), científicamente (universidades y centros de investigación) y de forma mixta, lo que produce un efecto positivo en su desempeño innovador. Asismismo, Urbano et al. (2018), quienes confirman la relevancia de un entorno de apoyo para desarrollar innovación tecnológica y emprendimiento innovador, ya que algunas condiciones reguladoras (derechos de propiedad y programas gubernamentales) benefician esta actividad, mientras que las relativas a ciencia y tecnología, así como condiciones normativas (percepción de oportunidades y cultura nacional) retardan el desarrollo de emprendimientos tecnológicos.

La tercera pregunta se formuló a partir de los trabajos de Guerrero y Urbano (2017), así como de Bautista (2014). Si bien en el primero se analizaron los efectos producidos por los vínculos con la triple hélice respecto a su desempeño innovador, y los efectos de moderación generados por empresas innovadoras y tradicionales, los autores proponen utilizar en futuras investigaciones distintas técnicas que permitan 
explorar el impacto regional. En el segundo se habla sobre la importancia de la vinculación triple hélice para el desarrollo y crecimiento económico de México, ya que, aunque se encontró que el gobierno está impulsando esta actividad, no se profundiza en la dinámica de colaboración en el contexto regional (a nivel estado), ni desde la perspectiva de los agentes.

Por último, la cuarta y quinta preguntas se elaboraron para enriquecer los estudios de Dosal et al. (2011), Malecki (2018) y Ferreira et al. (2016). En el primero identificaron que las empresas innovadoras utilizan el desarrollo tecnológico colaborativo como ventaja comparativa, y proponen indagar temas como capital social, educación, entre otros, para impulsar esta actividad. Mientras que en el segundo, basándose en los resultados de una revisión de literatura, invitan a realizar investigación comparativa del ecosistema emprendedor para empresas tecnológicas en varias etapas de desarrollo, así como de los beneficios de las redes sociales, conexiones, diversidad de empresas y subsistemas. Finalmente, en el tercero, derivado de un análisis bibliométrico, se plantea estudiar el efecto directo de los diferentes actores involucrados en el proceso del emprendimiento tecnológico.

Una vez que se obtuvo la versión final de la guía y estructura de la herramienta (Salinas y Be, 2019), se buscó a los informantes clave mediante la selección de la muestra cualitativa para poder realizar la entrevista, la cual se llevó a cabo de manera personal y presencial en cuatro de los siete casos, mientras que a los tres restantes se les envió mediante correo electrónico debido a su poca disponibilidad de tiempo. No obstante, a pesar de la situación, las respuestas obtenidas fueron muy completas y satisfactorias al contener la descripción de la visión de cada uno.

De acuerdo con Strauss y Corbin (2002), el propósito del muestreo teórico es acudir a lugares, personas o acontecimientos que representen una oportunidad para descubrir nuevas variaciones entre conceptos, de los cuales se puede obtener información con un respaldo teórico. La selección de la muestra para esta investigación se realizó a conveniencia, debido a la accesibilidad y proximidad de los entrevistados (López, 2004; Otzen y Manterola, 2017); se eligieron a siete sujetos de estudio representantes de cada actor de la cuádruple hélice (Cuadro 3). 
El papel de los actores de la cuádruple... González T., A.; J. Lavín V. y N.A. Pedraza M.

\section{CUADRO 3}

DATOS SOBRE LOS ENTREVISTADOS

\begin{tabular}{|c|c|c|}
\hline Código & Cargo & Organismo \\
\hline ENT01 & $\begin{array}{l}\text { Director de proyectos estratégicos para la pequeña } \\
\qquad y \text { mediana empresa }\end{array}$ & $\begin{array}{c}\text { Secretaría de Desarrollo Económico de } \\
\text { Tamaulipas }\end{array}$ \\
\hline ENT02 & $\begin{array}{l}\text { Jefe del departamento de patentes y transferencia } \\
\text { tecnológica }\end{array}$ & $\begin{array}{l}\text { Consejo Tamaulipeco de Ciencia y } \\
\text { Tecnología }\end{array}$ \\
\hline ENT03 & Rector & Universidad Politécnica de Victoria \\
\hline ENT04 & Director general & Quomnium (empresa consultora) \\
\hline ENT05 & $\begin{array}{l}\text { Directora del departamento de gestión tecnológica } \\
\qquad y \text { vinculación }\end{array}$ & Instituto Tecnológico de Ciudad Victoria \\
\hline ENT06 & Director de Desarrollo Profesional & Universidad Autónoma de Tamaulipas \\
\hline ENT07 & Programa Universitario Emprendedor & Universidad Autónoma de Tamaulipas \\
\hline
\end{tabular}

Fuente: elaborado a partir de Ferreira et al. (2016).

Como se observa en el Cuadro 3, los entrevistados ocupan cargos directivos o gerenciales que les permiten tener un panorama general del organismo, y se encargan de buscar y realizar la vinculación con otros agentes. Además, el entrevistado ENT01 cuenta con amplia experiencia en puestos gerenciales de ventas en la iniciativa privada, mientras que ENT02 ha ocupado puestos de protección industrial y transferencia de conocimiento, en administración pública y universidad por más de 20 años. Por otra parte, el entrevistado ENT03, además de ejercer en el sector educativo por casi una década, ha desempeñado cargos gerenciales en distintas empresas. A su vez, ENT04 combina sus actividades empresariales con la docencia. Los entrevistados ENT05, ENT06 y ENT07 son académicos reconocidos por su desempeño y labor administrativa enfocada en el desarrollo de competencias emprendedoras y su aplicación en el contexto empresarial real.

Una vez recopilada la información por medio de las entrevistas, se procedió a la transcripción para su procesamiento mediante un análisis de contenido cualitativo, técnica que permite la validez e interpretación de textos y documentos de forma explícita (lo expresado directamente) e implícita (lo que se pretende expresar), considerando no sólo el contenido, sino también el contexto, a través de procedimientos de descomposición y clasificación (Díaz, 2018; Saunders et al., 2009). Para ello se 
utilizó el Software MAXQDA Analytics, herramienta tecnológica para el análisis de datos cualitativos (VERBI GmbH, 2020).

\section{FOMENTO AL EMPRENDIMIENTO TECNOLÓGICO DESDE LA PERSPECTIVA DE LOS AGENTES DE LA CUÁDRUPLE HÉLICE EN TAMAULIPAS}

Como se ha mencionado, con este estudio se pretende conocer el papel de cada actor de la cuádruple hélice en el emprendimiento tecnológico de Tamaulipas desde su perspectiva, para lo cual se entrevistaron siete representantes de cada una de las hélices, lo que generó información cualitativa que fue procesada mediante un software especializado. Una vez que se importaron las transcripciones de las entrevistas al programa MAXQDA, se trabajó en la edición del documento, para hacerlo más amigable a la hora de interpretar la información.

Posteriormente se realizó una búsqueda de frecuencias para la elaboración de una lista de exclusión de palabras que no se utilizarían y una lista de inclusión de las palabras más importantes para este trabajo, las cuales se muestran en la Gráfica 1. Al realizar este ejercicio, se procuró disminuir la posibilidad de que términos irrelevantes o no relacionados con el objetivo de estudio fueran considerados en los hallazgos, y a su vez, que al incluir sólo los más importantes o recurrentes se obtuviera información útil para poder responder efectivamente a la pregunta de investigación.

GRÁFICA 1

FRECUENCIA DE PALABRAS DE LA LISTA DE INCLUSIÓN

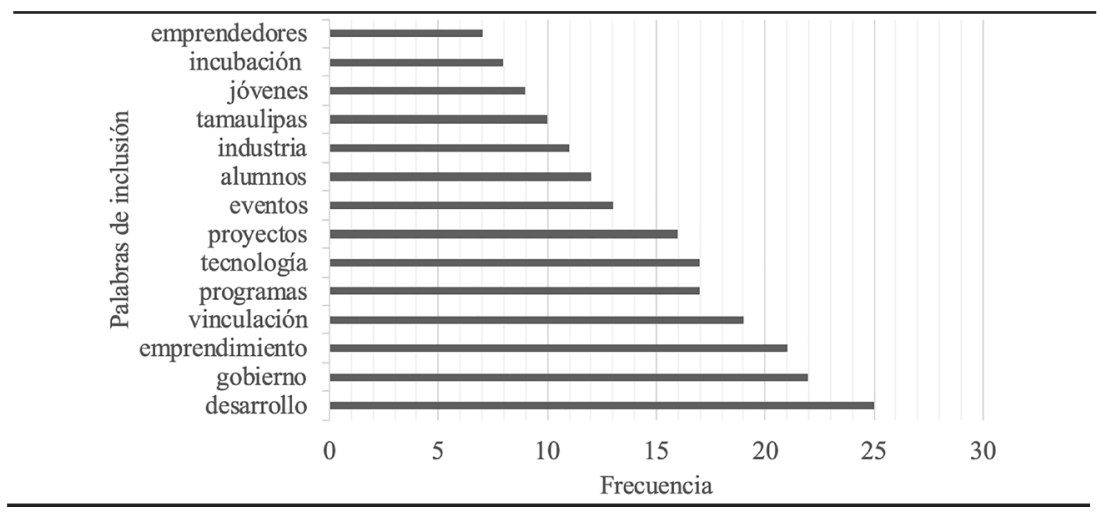

Fuente: elaboración propia. 
Como se observa, entre las palabras más frecuentes se encuentran: desarrollo, gobierno, emprendimiento, vinculación, programas, tecnología, proyectos, entre otras relacionadas con el objetivo de estudio, lo que facilitó el análisis y la interpretación, por lo que a partir de éstas se realizó la codificación que, de acuerdo con Miles et al. (2013) son códigos asignados inicialmente a los fragmentos de datos e incluyen hasta 25 enfoques diferentes, cada uno con una función o propósito particular.

En esta investigación se utilizó la codificación descriptiva, ya que asigna etiquetas a los datos para resumirlos en una palabra o frase corta (Díaz, 2018). Primero, se seleccionaron los dos conceptos más importantes de la indagación, la cuádruple hélice y el emprendimiento tecnológico. A partir de esto, se identificaron las palabras que más se relacionaban con estos temas, así como con las preguntas de la entrevista y se convirtieron en códigos, los cuales se agruparon para poder visualizar su representatividad, y a su vez se agregaron subcódigos para obtener una mejor descripción de éstos, lo que se conoce como sistema de codificación, que permite una mejor visión de los resultados del análisis de contenido, como se expone en la Figura 2.

En la Figura 2 se presenta el sistema de codificación del emprendimiento tecnológico descrito por los entrevistados como un medio por el cual las personas pueden "desarrollar sus propias empresas a través de las diferentes técnicas de tecnología que desarrollan en las mismas universidades [...]" (ENT01), además de ser "un tema que va en auge [...]" (ENT01), que cuenta con mucho apoyo gubernamental y para lo cual se trabaja en conjunto desde diversos sectores. No obstante, en cuanto al estado actual, lo perciben con poco avance, principalmente al concientizar a los jóvenes de optar por este tipo de emprendimiento, ya que "se encuentra en desarrollo y crecimiento, por tanto, no está consolidado [...]" (ENT06). 
FIGURA 2

CODIFICACIÓN DEL EMPRENDIMIENTO TECNOLÓGICO

\begin{tabular}{rll}
\hline Código & \multicolumn{1}{c}{ Concepto } \\
\hline P1-EA & Estado actual \\
P2-DE-PC & Desafios & Problema de comercialización \\
P2-DE-IN & Infraestructura \\
P2-DE-AP & Agenda pública \\
P2-DE-FI & Falta de información \\
P2-DE-FA & Falta de apoyo económico \\
P2-DE-CO & Comunicación \\
P3-ES & Estrategias \\
P3-ES-CN & Congresos \\
P3-ES-TT & Transferencia tecnológica \\
P3-ES-VN & Vinculación \\
P3-ES-FE & Ferias o exposiciones \\
P3-ES-DT & Desarrollo tecnológico \\
P3-ES-AI & Atracción e inversión \\
P4-IM & Impulso o fomento \\
P4-IM-AE & Apoyos económicos \\
P4-IM-IN & Incubación \\
P4-IM-CO & Concursos \\
P4-IM-TA & Talleres \\
P4-IM-DT & Desarrollo tecnológico \\
P4-IM-EV & Eventos
\end{tabular}

Fuente: elaboración propia.

En este mismo orden de ideas, los entrevistados expusieron su intención de poner a Tamaulipas en la mira "no solamente en el resto del país, sino también en el mundo [...]" (ENT01) en materia de innovación y desarrollo tecnológico, por lo que se requiere centrarse en los desafíos que esto representa, como hacer llegar a los jóvenes emprendedores la información sobre los programas y convocatorias que existen, ya que, si bien se han visto disminuidos a nivel federal en los últimos años, son importantes para el cumplimiento de objetivos de crecimiento y consolidación empresarial, por lo que es necesario mejorar la comunicación con los interesados.

Además, la falta de información sobre las herramientas de financiamiento y "conocimiento de lo que implica la tecnología [...]" (ENT04) para el crecimiento, lo que deriva en la necesidad de "promover el ecosistema de emprendimiento de empresas con base tecnológica" (ENT06), 
así como enfocar la atención hacia el capital humano y una "prospectiva laboral con enfoque en tecnologías o industria 4.0" (ENT06). Lo que deriva en la urgencia por incluirlo en la agenda pública, al ser un tema de interés real, que requiere un "mayor enfoque y esfuerzo por parte de organismos públicos [...] para fomentar y hacer crecer el emprendimiento tecnológico" (ENT06).

Otro reto es una infraestructura adecuada para el emprendimiento tecnológico, por lo que se requiere la creación de "institutos o centros de investigación y desarrollo públicos [...] o privados, así como de universidades e instituciones educativas con enfoque en tecnología" (ENT06), en donde se dote a los nuevos empresarios de infraestructura y servicios básicos gratuitos para desarrollar su proyecto, lo que beneficiaría la comercialización de innovación, toda vez que "la colocación de un producto o servicio tecnológico no es tarea fácil” (ENT07), como tampoco "establecer o lograr un acuerdo de licencia con un gran fabricante o distribuidor, que tenga los recursos y/o vínculos" (ENT07) para hacerlo.

Al respecto, los entrevistados propusieron estrategias para desarrollar un ecosistema de emprendimiento tecnológico, mediante la creación de mecanismos de incubación para "atender las necesidades regionales [...]" (ENT03), en donde se realicen "guías, talleres o cursos para concientizar a quien desee emprender, cómo iniciar y consolidar su empresa, ya sea de corte industrial, tecnológico o de servicio" (ENT05). Así como, llevar a cabo congresos, fomentar la transferencia de tecnología, "una vinculación real [...]" (ENT02) en la que exista un diálogo permanente entre los agentes de la cuádruple hélice, la creación de más ferias o exposiciones, el impulso al desarrollo tecnológico, la apropiación intelectual y la atracción de inversión.

Además de esto, los entrevistados expresaron la necesidad de impulsar actividades de formación y capacitación para aumentar la innovación mediante la vinculación universidad-empresa-gobierno, para lo que proponen más apoyos económicos para la creación de más empresas de base tecnológica tanto estatales como federales, así como programas de incubación, más concursos de creatividad y robótica, talleres y cursos prácticos "para guiar, concientizar e impulsar el emprendimiento" (ENT05), así como la "protección intelectual, licenciamiento y aprovechamiento de fondos para desarrollo tecnológico" (ENT07). 
Entre otras actividades que sugieren impulsar está la generación de conocimiento para el desarrollo de nuevas tecnologías por parte de los emprendedores, así como eventos organizados por el sector empresarial u otros interesados, en los que los alumnos, nuevos empresarios y académicos "expongan y concursen con proyectos de emprendimiento tecnológico [...]" (ENT07) y a donde pueda asistir la sociedad, personal de gobierno y miembros de la industria, con el fin de propiciar la vinculación.

Por último, proponen mejorar la infraestructura de las universidades mediante la creación de más laboratorios y equipos especializados para que los alumnos hagan sus prácticas, así como "convocatorias con reglas de operación bien definidas, para apoyar el impulso al emprendimiento" (ENT05), dirigidas a la comunidad universitaria "para atender demandas tecnológicas del sector productivo" (ENT07), además de crear "un sistema de vigilancia estratégica del sector tecnológico, para efectos de identificar las áreas estratégicas de investigación y tecnologías emergentes en las que se concentrarán los esfuerzos de inversión para obtener los mayores beneficios económicos y sociales" (ENT06).

Aunado a lo anterior, las respuestas de los entrevistados permitieron encontrar si existe o no una vinculación entre los diversos actores de la cuádruple hélice (Figura 3). Comentaron que sí se lleva a cabo, ya que se busca mayor participación de los agentes; se requiere "el apoyo de todos, no nada más de gobierno, también del mismo sector empresarial, de las universidades [...]" (ENT01), y además incluir a la federación en los esfuerzos por fomentar esta práctica. Asimismo, se encontró una deficiencia en la comunicación de convocatorias y programas, para lo que proponen más compromiso de las áreas encargadas de esta tarea.

Como se muestra en la Figura 3, los representantes de la universidad hablaron sobre su función de atender las necesidades regionales a través de programas académicos, desarrollo de proyectos, programas de emprendimiento e incubación que ayudan a la formación académica de los estudiantes con base en la instrucción teórica, lo que concuerda con lo dicho por Afonso et al. (2012). En este sentido, la vinculación con los demás actores se da mediante estancias y estadías de los alumnos, además de las sinergias que se crean con centros de transferencia, tanto locales como nacionales, y las "alianzas con empresas que pueden generar apoyos para desarrollar los proyectos tecnológicos" (ENT07). 
FIGURA 3

CODIFICACIÓN DEL EMPRENDIMIENTO TECNOLÓGICO

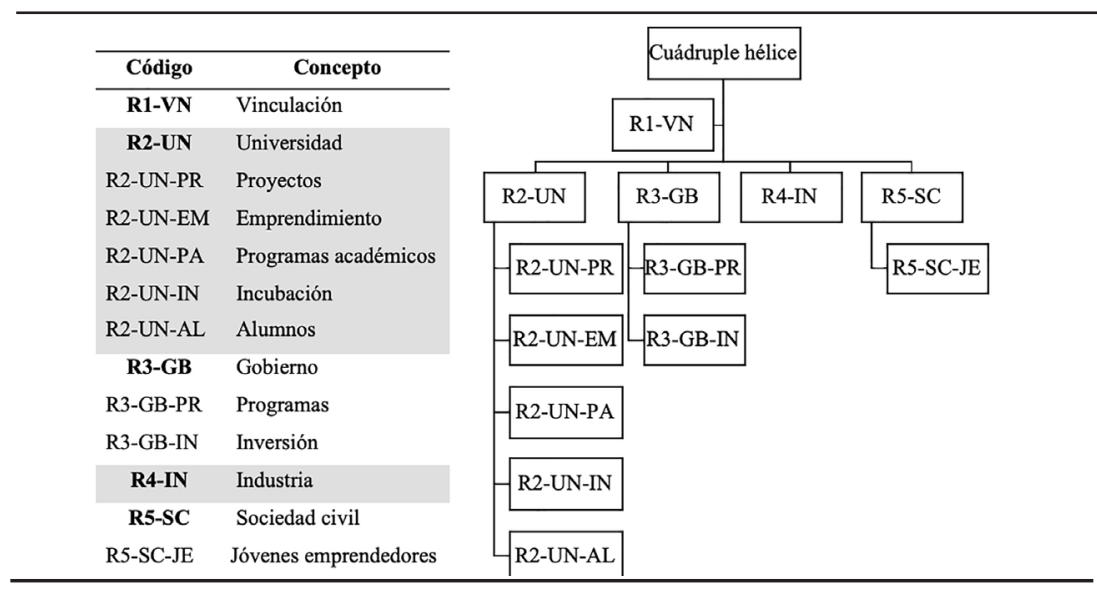

Fuente: elaboración propia.

Respecto a la forma en que la universidad fomenta el emprendimiento tecnológico, se identificó la realización de proyectos, inclusión del emprendimiento en los programas académicos, y actividades de incubación, todo enfocado a los alumnos. En este sentido, el "desarrollo de investigación y proyectos innovadores a través de la vinculación universidad-empresa-gobierno" (ENT06) lo realizan los alumnos de licenciatura, maestría o doctorado, así como catedráticos, con el fin de obtener información sobre las necesidades reales de la industria, y realizar una retroalimentación de los resultados.

Cabe agregar que la universidad considera el emprendimiento como elemento fundamental en la formación de sus estudiantes, por lo que se puede encontrar dentro de los planes de estudio, además de ofrecer programas de incubación de empresas, o bien asesoría, talleres, apoyos y espacios para el desarrollo de nuevas ideas, así como para la comercialización del bien o servicio. Por último, los entrevistados destacaron que los alumnos son la materia prima de las instituciones educativas, ya que "donde realmente nace la innovación es la imaginación de los alumnos [...]" (ENT03); a través de ellos se logra obtener ideas más innovadoras y mediante su formación académica se pueden convertir en los futuros emprendedores de base tecnológica.

Por otra parte, los representantes de gobierno indicaron que son diversas las maneras en que se vinculan con otros actores de la 
cuádruple hélice, ya sea involucrando a la universidad y centros de investigación en eventos que fomentan el emprendimiento e innovación, así como con otras dependencias públicas para financiar esta actividad, a través de la cual se crean relaciones con la industria. Esto complementa lo encontrado por Afonso et al., (2012) y Galvão et al. (2017), al incorporar la parte de los eventos y asistencia entre diversas entidades gubernamentales.

En este sentido, el gobierno apoya el emprendimiento tecnológico a través de diversos programas que asignan recurso, capacitación e incluso espacios para la exposición de proyectos, así como para cubrir gastos de protección intelectual e incubación; también se encargan de buscar una mayor inversión en empresas locales, para el desarrollo de nuevas tecnologías e innovación. Sobre los programas que ofrecen, además del CECIT y Creciendo con tu empresa, de Cotacyt, se pudo identificar en las respuestas de los entrevistados el programa Tamaulipas emprende, de la Secretaría de Desarrollo Económico del estado, y el Programa de Estímulos a la Innovación (PEI) del gobierno federal.

Respecto a la industria, los entrevistados afirman que se encuentra integrada por empresas que fungen como proveedoras de materias primas y como soporte para el emprendimiento tecnológico mediante asesorías o consultoría y financiamiento, y contribuyen a esta práctica a través de asociaciones empresariales. Si bien estos hallazgos concuerdan con la descripción del papel de las compañías como parte de la cuádruple hélice de Afonso et al. (2012) y Galvão et al. (2017), se pudo observar una participación débil del sector tanto en la práctica de la vinculación como en el fomento de la actividad emprendedora de base tecnológica.

En cuanto al papel de la sociedad civil, durante el presente ejercicio se observó que en Tamaulipas esta hélice se integra principalmente por jóvenes universitarios que muchas veces no cuentan con recursos económicos para emprender, y son a quienes se les debe considerar como prospectos para la creación de este tipo de empresas, ya que actualmente "sólo emprenden por necesidad [...]" (ENT05) y se enfocan en cubrir sus necesidades personales, por lo que dejan en segundo plano la inversión para el crecimiento de su empresa. Es de suma importancia identificarlos y hacerles saber que existen apoyos y herramientas para su emprendimiento. 
Es importante señalar que el papel de la sociedad civil como parte de la cuádruple hélice difiere de las descripciones realizadas por Afonso et al. (2012), Galvão et al. (2017), González (2019) y Guerrero y Urbano (2017), ya que éstos otorgan un rol más activo a las colaboraciones con los demás agentes, especialmente al considerarlas un eje central que crea un ambiente propicio para el emprendimiento tecnológico, con una demanda de productos y servicios innovadores y de mayor calidad, por lo que representan un área de oportunidad para explotar mejor estas relaciones.

\section{CONCLUSIONES}

Como se mencionó en un principio, el objetivo de este trabajo fue conocer el papel que desempeñan cada uno de los actores de la cuádruple hélice en el emprendimiento tecnológico en Tamaulipas, con la finalidad de profundizar en la comprensión de este fenómeno desde la perspectiva de los diferentes agentes, para lo cual se realizaron entrevistas a representantes de cada una de las hélices para recolectar información relevante al respecto.

Se pudo observar que la vinculación se da entre universidad, gobierno e industria, sin lograr integrar a la sociedad civil de manera significativa en el proceso, además de que se realiza mediante el desarrollo de proyectos colaborativos, eventos, estancias y estadías de alumnos. De esta manera, se identificaron áreas de oportunidad en la creación de sinergias, sobre todo al impulsar el emprendimiento tecnológico, ya que resulta necesario incorporar tanto al gobierno federal como a la sociedad civil.

Por otra parte, la investigación tiene algunas limitaciones derivadas de su diseño metodológico, debido a que se realizó en un periodo reducido y en un contexto específico (Tamaulipas, México), e involucró a un grupo particular de agentes (de la cuádruple hélice) de distintos sectores y actividades (industria, universidad, gobierno y sociedad civil). Además, se estudió una pequeña muestra de personas, lo cual puede limitar la capacidad de generalizar los resultados. No obstante, estas limitaciones son generales y algunos trabajos previos se realizaron bajo condiciones similares (Kleine et al., 2019; Roman et al., 2020; Vallance et al., 2020), por tanto, la utilidad del enfoque utilizado y la 
generalización de los hallazgos se pueden tomar como base en estudios posteriores que involucren a otros grupos de entrevistados.

Derivado de lo anterior, se propone para futuras investigaciones realizar estudios que comprueben cuantitativamente lo sugerido en la presente investigación, a partir de los hallazgos, utilizando escalas Likert, ya que se demuestra la importancia de estos conceptos en la actualidad en cuestión de desarrollo económico, que pueden dar pie a la construcción de estrategias de los agentes para una mejor vinculación y mejores resultados de indicadores de innovación, además de permitir la replicación en otros contextos. También se propone dar continuidad al presente mediante el análisis del desempeño innovador de los emprendimientos tecnológicos como resultado de las acciones de la cuádruple hélice para su desarrollo.

\section{Bibliografía}

Aarsand, L. y P. Aarsand (2018), "Framing and switches at the outset of qualitative research interviews", Qualitative Research, 19(6), pp. 635-652. $<$ doi:10.1177/1468794118816623>.

Afonso, O., S. Monteiro y M. Thompson (2012), "A growth model for the quadruple helix", Journal of Business Economics and Management, 13(5), pp. 849-865. <doi:10.3846/16111699.2011.626438>.

Afzal, M. N., D. D. Mansur, S. Siddiqui y J. Gope (2018), "A panel investigation of the triple helix $(\mathrm{TH})$, quadruple helix $(\mathrm{QH})$ relationship in ASEAN-5 economies", Journal of Innovation Economics y Management, 3(27), pp. 97-122. < doi:10.3917/jie.027.0097>.

Arenal, A., C. Armuña, C. Feijoo, S. Ramos, Z. Xu y A. Moreno (2020), "Innovation ecosystems theory revisited: The case of artificial intelligence in China", Telecommunications Policy, 44(6), pp. 1-27. <doi: 10.1016/j. telpol.2020.101960>.

Asociación Triple Hélice (2020), "Updates from the conference sessions", Triple Helix Conference 2020, 15-17 de junio. <https://events.tuni.fi/ thc2020/category/updates-from-the-conference-sessions/>.

Autio, E., M. Kenney, P. Mustar, D. Siegel y M. Wright (2014), "Entrepreneurial innovation: The importance of context", Research Policy, 43(7), pp. 1097-1108. <doi:10.1016/j.respol.2014.01.015>.

Banco Mundial (2020), "Gasto en investigación y desarrollo (\% del PIB)". $<$ https://datos.bancomundial.org/indicador/GB.XPD.RSDV.GD.ZS

Bărbulescu, O. y C.P. Constantin (2019), "Sustainable Growth Approaches: Quadruple Helix Approach for Turning Brașov into a Startup City", Sus- 
tainability, 11(21), pp. 1-19. < doi:10.3390/su11216154>.

Baumol, W. J. (1989), "Entrepreneurship in economic theory", American Economic Review Papers and Proceedings, 58(2), pp. 64-71.

Bautista, E. G. (2014), "La importancia de la vinculación universidad-empresa-gobierno en México", RIDE Revista Iberoamericana para la Investigación y el Desarrollo Educativo, 5(9), 0. <http://www.redalyc.org/articulo. oa? id=498150317008>.

Beckman, C., K. Eisenhardt, S. Kotha, A. Meyer y N. Rajagopalan (2012), "Technology entrepreneurship", Strategic Entrepreneurship Journal, 6, pp. 89-93. < doi:10.1002/sej.1134>.

Benavides, Ó. A. (2004), "La innovación tecnológica desde una perspectiva evolutiva", Cuadernos de Economía, 23(41), pp. 49-70.

Borghys, K., Sh. van der Graaf, N. Walravens y M. Van Compernolle (2020), "Multi-Stakeholder Innovation in Smart City Discourse: Quadruple Helix Thinking in the Age of 'Platforms"'. Frontiers in Sustainable Cities, 2, pp. 1-6. <doi: 10.3389/frsc.2020.00005>.

Byankin, A. S. y G.I. Burdakova (2019), "Formation of competences of technology entrepreneurship based on the 'triple helix' model", St. Petersburg State Polytechnical University Journal. Economics, 12(3), pp. 187-199. <doi: 10.18721/JE.12316>.

Cadena-Iñiguez, P., R. Rendón-Medel, J. Aguilar-Ávila, E. Salinas-Cruz, F.d. de la Cruz-Morales y D.M. Sangerman-Jarquín (2017), "Métodos cuantitativos, métodos cualitativos o su combinación en la investigación: un acercamiento en las ciencias sociales", Revista Mexicana de Ciencias Agricolas, 8(7), pp. 1603-1617. <http://www.redalyc.org/articulo. oa? id=263153520009>.

Canales, R. A., Y. G. Román y W. Ovando (2017), "Emprendimiento de la población joven en México. Una perspectiva crítica”, Entreciencias: diálogos en la Sociedad del Conocimiento, 5(12), pp. 1-23. <doi:10.21933/J. EDSC.2017.12.211>.

Carayannis, E. G. y D.F. Campbell (2009), “'Mode 3' and 'Quadruple Helix': toward a 21st century”, Int. J. Technology Management, 46(3/4), pp. 201234.

Castillo, L., J. Lavín y N.A. Pedraza (2014), “La gestión de la triple hélice: fortaleciendo las relaciones entre la universidad, empresa, gobierno". Multiciencias, 14(4), pp. 438-446. <https://www.redalyc.org/articulo. oa? id $=904 / 90433839002>$.

Castillo, E., y M.L. Vásquez (2003), "El rigor metodológico en la investigación cualitativa", Colombia Médica, 34(3), pp. 164-167. <http://www.redalyc.org/articulo.oa?id=28334309>.

Consejo Nacional de Ciencia y Tecnología (Conacyt) (2019), "Programa Estratégico Nacional de Tecnología e Innovación Abierta (PENTA)". < https:// www.conacyt.gob.mx/index.php/el-conacyt/penta> [29 de enero de 2020]. 
Da Costa, A. A., D. Leandro, K. Carvalho, C. Carvalho y M.J. de Brito (2018), "A systematic review from triple to quintuple helix", Ey G Economia e Gestão, 18(51), pp. 77-93. <doi:10.5752/P.1984-6606.2018v18n51p77-93>.

Díaz, C. (2018), "Investigación cualitativa y análisis de contenido temático. Orientación intelectual de revista Universum", Revista General de Información y Documentación, 28(1), pp. 119-142. <doi:10.5209/ RGID.60813>.

Dosal, C., C.I. Gutiérrez y A. Saracho (2011), “¿Quiénes son los emprendedores innovadores mexicanos?”. Fundación Idea, México. <https://website-c230-consultores.nyc3.digitaloceanspaces.com/IDEA/files/Emprendedores_Mexicanos_1533334895.pdf>. [26 de agosto de 2019].

De Dushnitsky, G. y M.J. Lenox (2005), "When do incumbents learn from entrepreneurial ventures? Corporate venture capital and investing firm innovation rates", Research Policy, 34(5), pp. 615-639. <doi:10.1016/j. respol.2005.01.017>.

Duval-Coueti, N., M. Ladisch y S. Yi (2020), “Addressing academic researcher priorities through science and technology entrepreneurship education", Journal of Technology Transfer. <doi: 10.1007/s10961-020-09787-5>.

Etzkowitz, H. y L. Leydesdorff (1995), "The triple helix university-industrygovernment relations: A laboratory for knowledge based economic development", EASST Review, 14(1), pp. 14-19.

Fernández, J. (2015), “Economía neo-schumpeteriana, innovación y política tecnológica", Cuadernos de economía, 38, pp. 79-89. < doi:10.1016/j.cesjef.2015.03.001>.

Ferreira, J. J., F.A. Ferreira, C.I. Fernandes, M.S. Jalali, M.L. Raposo y C.S. Marques (2016), "What do we [not] know about technology entrepreneurship research?", International Entrepreneurship and Management Journal, 12(3), pp. 713-733. >doi:10.1007/s11365-015-0359-2>.

Galvão, A. R., C. Mascarenhas, R. Rodrigues, C. Marques y C. Leal (2017), "A quadruple helix model of entrepreneurship, innovation and stages of economic development", Review of International Business and Strategy, 27(2), pp. 261-282. <doi:10.1108/RIBS-01-2017-0003>.

Galvão, A., C. Mascarenhas, C. Marques, J. Ferreira y V. Ratten (2019), “Triple helix and its evolution: a systematic literature review", Journal of Science and Technology Policy Management, 10(3), pp. 812-833. < doi: 10.1108/ JSTPM-10-2018-0103>.

Garud, R. y P. Karnø (2003), "Bricolage versus breakthrough: distributed and embedded agency in technology entrepreneurship", Research Policy, 32(2), pp. 277-300. <doi:10.1016/S0048-7333(02)00100-2>.

Godin, B. (2008), "In the Shadow of Schumpeter: W. Rupert Maclaurin and the Study of Technological Innovation", Minerva, 46, pp. 343-360. $<$ doi:10.1007/s11024-008-9100-4>.

González, A. (2019), "Puntos de convergencia para la vinculación cuádruple 
hélice", en Tamaulipas: Foro estatal de consulta "humanidades, ciencia y tecnología en México: presente y futuro", 21 de marzo. Consejo Tamaulipeco de Ciencia y Tecnología Ciudad Victoria, Tamaulipas, México. http://plataforma.cotacyt.gob.mx/forotamaulipas/ponencias/02-13.pdf. [junio de 2019].

Guerrero, M., y D. Urbano (2017), "The impact of Triple Helix agents on entrepreneurial innovations' performance: An inside look at enterprises located in an emerging economy", Technological Forecasting y Social Change, 119, pp. 294-309. <doi:10.1016/j.techfore.2016.06.015>.

Hamui, A. (2016), "La pregunta de investigación en los estudios cualitativos", Investigación en educación médica, 5(17), pp. 49-54. < doi:10.1016/j. riem.2015.08.008>.

Hasche, N., L. Höglund y G. Linton (2019), "Quadruple helix as a network of relationships: creating value within a Swedish regional innovation system", Journal of Small Business \& Entrepreneurship, pp. 1-22. <doi: 10.1080/08276331.2019.1643134>.

Hernández, R., C. Fernández y P. Baptista (2003), Metodología de la investigación. México: McGraw-Hill Interamericana. 3. ${ }^{a}$ ed.

Höglund, L. y G. Linton (2018), "Smart specialization in regional innovation systems: a quadruple helix perspective", $R$ and D Management, 48(1), pp. 60-72. <doi:10.1111/radm.12306>.

Iqbal, J., S. Kousar y W. ul Hameed (2018), “Antecedents of sustainable social entrepreneurship initiatives in Pakistan and Outcomes: Collaboration between quadruple helix sectors", Sustainability, 10(12), pp. 1-21. < doi: 10.3390/su10124539>.

Kleine, K., F. Giones y S. Tegtmeier (2019), "The Learning Process in Technology Entrepreneurship Education-Insights from an Engineering Degree”, Journal of Small Business Management, 57(S1), pp. 94-110. < doi: $10.1111 / \mathrm{jsbm} .12514>$.

Leydesdorff, L. (2012), “The Triple Helix, Quadruple Helix, ..., and an NTuple of Helices: Explanatory Models for Analyzing the KnowledgeBased Economy?", Journal of the Knowledge Economy, 3(1), pp. 25-35. $<$ doi:10.1007/s13132-011-0049-4>.

López, O., M. Blanco y S. Guerra (2009), "Evolución de los modelos de la gestión de innovación", Innovaciones de negocios, 5(2). $<$ http://revistainnovaciones.uanl.mx/index.php/revin/article/view/210>. [01 de mayo de 2019].

López, P. L. (2004), "Población muestra y muestreo", Punto Cero, 9(8), pp. 6974. $<$ http://www.scielo.org.bo/scielo.php?script=sci_arttextypid=S181502762004000100012ylng=esytlng=es $>$.

Malecki, E. J. (2018), "Entrepreneurship and entrepreneurial ecosystems", Geography Compass, 12(3), pp. 1-21. <http://doi.wiley.com/10.1111/ gec3.12359>. 
Marín, A. e I. Rivera (2014), "Revisión teórica y propuesta de estudio sobre el emprendimiento social y la innovación tecnológica", Acta Universitaria, 24(1), pp. 48-58. <http://www.redalyc.org/articulo.oa?id=41648308005>. [06 de mayo de 2019].

Marques, C., A.V. Marques, V. Braga y V. Ratten (2020), “Technological transfer and spillovers within the RIS3 entrepreneurial ecosystems: a quadruple helix approach", Knowledge Management Research and Practice, pp. 1-10. <doi:10.1080/14778238.2020.1777909>.

McAdam, M., K. Miller y R. McAdam (2016), “Understanding Quadruple Helix relationships of university technology commercialisation: a microlevel approach", Studies in Higher Education, 43(6), pp. 1058-1073. <doi: 10.1080/03075079.2016.1212328>.

Miles, M., M. Huberman y J. Saldana (2013), Qualitative data analysis. Thousand Oaks, SAGE Publications.

Miller, K., R. McAdam y M. McAdam (2016a), “A systematic literature review of university technology transfer from a quadruple helix perspective: toward a research agenda", RyD Management, 48(1), 7-24. < doi:10.1111/ radm.12228>.

Miller, K., R.McAdam, A. Alexander y P. Puthusserry (2016b), “Knowledge transfer in university quadruple helix ecosystems: an absorptive capacity perspective", RyD Management, 46, pp. 383-399. < doi:10.1111/ radm.12182>.

Moral, C. (2006), "Criterios de validez en la investigación cualitativa actual". Revista de Investigación Educativa, 24(1), pp. 147-164. <http://www.redalyc.org/articulo.oa?id=283321886008>.

Moreno, H. M., y R. Espíritu (2010), “Análisis de las características del emprendimiento y liderazgo en los países de Asia y Latinoamérica", PORTES, Revista mexicana de estudios sobre la Cuenca del Pacífico, 4(8), pp. 101-122.

Morgan, K. (1997), “The Learning Region: Institutions, Innovation and Regional Renewal”, Regional Studies, 31(5), pp. 491-503. < doi:10.1080/00 343409750132289>.

Morse, J. M., M. Barrett, M. Mayan, K. Olson y J. Spiers (2002), "Verification Strategies for Establishing Reliability and Validity in Qualitative Research", International Journal of Qualitative Methods, 1(2), pp. 13-22. $<$ doi:10.1177/160940690200100202>.

Mosey, S. (2016), "Teaching and research opportunities in technology entrepreneurship", Technovation, 57-58(2), pp. 43-44. <doi: 10.1016/j.technovation.2016.08.006>.

Mosey, S., M. Guerrero y A. Greenman (2017), “Technology entrepreneurship research opportunities: insights from across Europe", The Journal of Technology Transfer, 42(1), pp. 1-9. < doi:10.1007/s10961-015-9462-3>.

Otzen, T. y C. Manterola (2017), “Técnicas de Muestreo sobre una Pobla- 
ción a Estudio", International Journal of Morphology, 35(1), pp. 227-232. $<$ https://dx.doi.org/10.4067/S0717-95022017000100037>.

Parveen, S., A. A. Senin y A. Umar (2015), "Organization culture and open innovation: A quadruple helix open innovation model approach", International Journal of Economics and Financial Issues, 5(2), pp. 335-342.

Plaza, J. J., P.A. Uriguen y H. F. Bejarano (2017), "Validez y confiabilidad en la investigación cualitativa", ARJÉ, 11(21), pp. 352-357. <http://arje. bc.uc.edu.ve/arj21/art24.pdf $>$.

Riese, J. (2019), "What is 'access' in the context of qualitative research?", Qualitative Research, 19(6), pp. 669-684. <doi:10.1177/1468794118787713>.

Roman, M., H. Varga, V. Cvijanovic y A. Reid (2020), "Quadruple Helix Models for Sustainable Regional Innovation: Engaging and Facilitating Civil Society Participation", Economies, 8(2), pp. 1-15. <doi: 10.3390/economies8020048>.

Salgado, A. C. (2007), "Investigación cualitativa: diseños, evaluación del rigor metodológico y retos", Liberabit, 13(13), pp. 71-78.

Salinas, C. y P. A. Be (2019), "Entrevista a profundidad", en A. I. Máynez Guaderrama y V. G. López Torres, La práctica de la investigación en las ciencias administrativas. Pearson, México. pp. 79-92.

Sánchez, Y., F. García y E. Mendoza (2014), "Determinantes de la capacidad de innovación regional en México. Una tipología de las regiones", Región y sociedad, 26(61), pp. 119-160. <doi:10.1016/j.estger.2015.04.001>.

Saunders, M., P. Lewis y A. Thornhill (2009), Research methods for business students. Prentice Hall, Londres. 5. ${ }^{\mathrm{a}}$ ed.

Schumpeter, J. (1934), The Theory of Economic Development. Harvard University Press, EUA.

Schütz, F., M.L. Heidingsfelder y M. Schraudner (2019), "Co-shaping the future in quadruple helix innovation systems: uncovering public preferences toward participatory research and innovation", She Ji: The Journal of Design, Economics, and Innovation, 5(2), pp. 128-146. < doi: 10.1016/j. sheji.2019.04.002>.

Secretaría de Economía (2016), "PROSOFT INDUSTRIA 4.0 MX". México. $<$ https://prosoft.economia.gob.mx/acercade/>. [29 de enero de 2020].

Strauss, A. y J. Corbin (2002), Bases de la investigación cualitativa: técnicas y procedimientos para desarrollar la teoría fundamentada. Medellín: Universidad de Antioquia.

Suzic, B., A. Ulmer y J. Schumacher (2020), "Complementarities and Synergies of Quadruple Helix Innovation Design in Smart City Development", Smart City Symposium Prague (SCSP), 25 de junio. Praga, República Checa. $<$ doi:10.1109/SCSP49987.2020.9133961>.

Symon, G. y C. Cassell (2004), Essential guide to qualitative methods in organizational research. SAGE Publications, Londres.

Urbano, D., M. Guerrero, J.J. Ferreira y C.I. Fernandes (2018), “New tech- 
nology entrepreneurship initiatives: Which strategic orientations and environmental conditions matter in the new socio economic landscape?", The Journal of Technology Transfer, pp. 1-26. < doi:10.1007/s10961-0189675-3>.

Vallance, P., M. Tewdwr-Jones y L. Kempton (2020), "Building collaborative platforms for urban innovation: Newcastle City Futures as a quadruple helix intermediary", European Urban and Regional Studies, pp. 1-17. <doi: $10.1177 / 0969776420905630>$.

Venkataraman, S. (2004), "Regional transformation through technological entrepreneurship", Journal of Business Venturing, 19(1), pp. 153-167. $<$ doi:10.1016/j.jbusvent.2003.04.001>.

VERBI GmbH (2020), "MAXQDA The Art of Data Analysis". <https:// es.maxqda.com>

Wheadon, M. y N. Duval-Couetil (2018), "Token entrepreneurs: a review of gender, capital, and context in technology entrepreneurship", Entrepreneurship and Regional Development, 31(3-4), pp. 308-336. <doi: 10.1080/08985626.2018.1551795>.

Xue, J. y P. G. Klein (2010), "Regional determinants of technology entrepreneurship", International Journal of Entrepreneurial Venturing, 1(3), pp. 291-308. < doi:10.1504/IJEV.2010.030978>.

Zahra, S. y M. Wright (2016), "Understanding the Social Role of Entrepreneurship", Journal of Management Studies, 53(4), pp. 610-629. < doi:10.1111/ joms.12149>.

Zavatta, R. (2008), Financing Technology Entrepreneurs y SmEs in Developing Countries: Challenges and Opportunities. InfoDev / World Bank, Washington, DC. $<$ http://www.infodev.org/infodev-files/resource/InfodevDocuments_542.pdf $>$. 